\title{
Fútbol como programa deportivo para menores con TEA en educación primaria
}

Football as a sports program for children with ASD in elementary education

O futebol como programa esportivo para crianças com TEA no ensino fundamental

\section{DOI: https://doi.org/10.18861/cied.2021.12.1.3065}

\section{José María López Díaz}

Universidad Rey Juan Carlos

España

josemaria.lopez@urjc.es

ORCID: 0000-0003-3595-5766

Ricardo Moreno Rodriguez

Universidad Rey Juan Carlos

España

ricardo.moreno@urjc.es

ORCID: 0000-0003-2593-4101

José Luis López Bastías

Universidad Rey Juan Carlos

España

joseluis.lopez@urjc.es

ORCID: 0000-0002-9454-5428

Recibido: 05/05/2020

Aprobado: 27/10/2020

Cómo citar: López Díaz, J. M., Moreno Rodríguez, R., \& López Bastías, J. L. (2021). Fútbol como programa deportivo para menores con TEA en educación primaria. Cuadernos de Investigación Educativa, 12(1). https://doi.org/10.18861/ cied.2021.12.1.3065

\section{Resumen}

Las dificultades que tienen los niños con Trastorno del Espectro Autista (TEA) para realizar ejercicio fisico y deportivo es una realidad. Esta situación provoca una baja participación en actividades fisicas y deportivas con sus iguales. Los problemas que presentan a nivel motor dificultan la participación en actividades fisicas grupales, provocando tambien un descenso en la sociatización dentro del propio contexto deportivo (Kruger et al, 2019). Por ello es necesario proponer programas orientados a la practica fisica y deportiva que favorezcan la participacion en actividades fisicas y un aumento de las relaciones sociales (Wong et al, 2015). Con la intención de promocionar el deporte para potenciar las habilidades fisicas y sociales con el fin de producir una mejora genoralizada en ambas $y$ de esta forma transferi sus

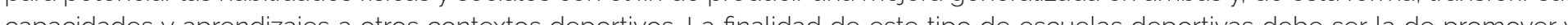

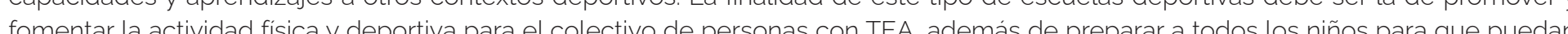
participar en cualquiertipo de deporte En ocasiones, la falta de información referente a la discapacidad puede provocar un rechazo hacia las personas con discapacidad por parte de los responsables encargados de dirivir la práctica deportiva buscando la intención enacia

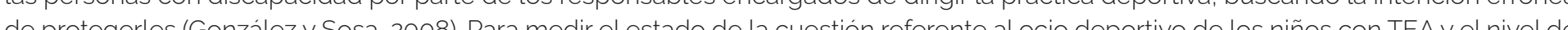
ale proteg resultados obtenidos al término de la investigación fueron positivos, lo que permitió justificar el impulso de la actividad fisica y deportiva como una alternativa de ocio para las personas con TEA

\section{Abstract}

The difficulties that childien wh Autism Spectrum Disorder (ASD) experience to perform physical and sports exercises is a realty. This situation causes a low participation in physical and sports activities

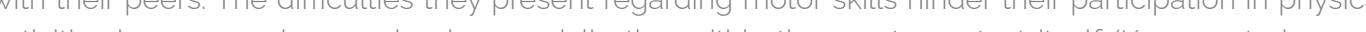
activities in groups, also causing less socialization within the sports context itself (Kruger, et al., 2019. herefore, it is nocessany to propose programis on wented to physical and spots practice, encouraging the participation in physical activities and increasing the social relations iwong et al, 2015). With the intention of promoting sports for this group, a sports tranining program was designed based on pre sports football games. The sessions managed to enhance childrens phiysical and social skits in order to attain a general purpose of this type of sports schus transter their capabiltes and teanning to ohner sports contexts. The purpe of the lack of information regarding disability can lead to a rejection of people with disabilities by those esponsible of directing sport practices, with the wrong intention of protecting them (González \& Sosa 2008). In order to measure the state of the art regarding sport leisure of children with ASD and the level of satisfaction reached under the sport training program, a questionnaire was distributed to the families of participants. The results obtained at the end of the research were positive, evidencing the need of promoting physical and sports activities as an alternative of leisure for people with ASD.

Keywords Autism Spectrum Disorder TEA, Disability, Physicat Activity Spot

\section{Resumo}

Euma realidade as dificuldades que meninos e meninas com Transtorno do Espectro do Autismo (TEA lêm para realizar exercicios fisicos e esportes. Essa situação acarreta baixa participação em atividades ísicas e esportivas com seus companheiros. Os problemas que apresentam no nivel motor dificultam a participação em atividades fisicas em grupo, causando também uma diminuição da socialização no próprio contexto esportivo (Kruger, et al., 2019). Portanto, é necessário propor programas voltados para prática física e esportiva que favoreçam a participação em atividades fisicas, bem como o incremento das elações sociais (Wong et al., 2015). Com o intuito de promover o esporte para esse grupo, foi elaborado m programa de treinamento esportivo baseado em jogos de futebol pré-esportivos. As sessões serviram para potencializar as aptidões fisicas e sociais de forma a produzir uma melhoria geral em ambas e, dessą maneira, transferir as suas competências e aprendizagem para outros contextos esportivos. A finalidade desse tipo de esporte nas escolas deve ser a promoção e o incentivo à atividade física e esportiva do grupo de pessoas com TEA, além de preparar todas as crianças para que possam praticar qualquer tipo de esporte. Ás vezes, a falta de informação sobre a deficiência pode causar uma rejeição por parte dos responsáveis pelo direcionamento da prática esportiva, às pessoas com deficiência, buscando ntenção equivocada de protegê-los (González e Sosa, 2008). Para mensurar o estado da arte em relaçâo a lazer esportivo de meninos e meninas com TEA e o nivel de satisfação atingido com o programa de reinamento esportivo, foi aplicado um questionário às familias dos participantes. Os resultados obtides a final da pesquisa foram positivos, o que permitiu justificar a promocão da atividade fisica e esportiva como alternativa de lazer para pessoas com TEA 


\section{Introducción}

El Trastorno del Espectro del Autismo (TEA) es considerado un trastorno del neurodesarrollo con una base biológica extremadamente compleja (DiCiccoBloom et al., 2006). Las personas diagnosticadas con TEA se caracterizan por presentar graves alteraciones permanentes en las principales áreas de desarrollo (Frith, 1989; Rivière, 1998), tener déficits persistentes en la comunicación e interacción social, conductas estereotipadas e intereses restringidos (American Psychiatric Association, 2013). Este tipo de patrones influye y limita la capacidad de elección para realizar cualquier tipo de actividad física o deportiva (American Psychiatric Association, 2000; Okely, Booth \& Patterson, 2001; Sallis, Prochaska \& Taylor, 2000). Esto supone un riesgo para la salud del niño pues las características propias que presenta dificultan la práctica deportiva, provocando el abandono de la práctica de la actividad física. Por este motivo es fundamental vincular el ejercicio físico a la vida diaria de este colectivo (Villalba, 2015) pues la inactividad puede provocar sedentarismo (Ruiz, Salinero, González, Lledó, Garcia y Theirs, 2015) y, por consiguiente, que se generen problemas de salud.

Debido a los diferentes niveles de severidad que presenta elTEAy a las necesidades individuales de cada persona, se hace necesario planificar programas flexibles e individualizados (Cuesta, Sánchez, Orozco, Valenti y Cottini, 2016). De acuerdo con Gutiérrez (2004) es importante promover el acceso a actividades físicas y deportivas teniendo en cuenta las características individuales de cada uno de los TEA.

Durante el devenir histórico, prácticamente hasta el momento presente, el colectivo de personas con TEA había sido apartado de cualquier tipo de actividad física y de ocio, pues todavía no existía una valoración positiva acerca de una práctica deportiva y el beneficio que esta podría aportar al trastorno. En cambio, para Julián, Abarca-Sos, Murillo y Aibar (2015), incluir la actividad física en la vida diaria de las personas con TEA supone un "impacto positivo en la salud mental y física, reduce los trastornos ambientales y comportamentales, promociona el desarrollo de la socialización, está dentro del foco de interés de los niños, niñas y adolescentes, e incrementa la satisfacción en la vida familiar" (p. 66). El ocio desempeña un papel importante en la vida personal de cada individuo. Todos los niños con TEA tienen derecho a disfrutar de cualquier tipo de ocio en su tiempo libre. Si, además, dentro de esta elección, puede incluirse la actividad fisica y deportiva, el beneficio puede ser mucho más amplio (Villalba, 2015).

Todas las personas con un diagnóstico TEA tienen la posibilidad real de aumentar su formación y su autonomía siempre y cuando los apoyos y servicios requeridos sean adecuados a las necesidades de la persona y tengan como objetivo el desarrollo continuo y progresivo (Confederación Autismo España, 2003). La situación y la evolución del trastorno vendrán marcadas por el tipo de atención recibida. Asimismo, si el tratamiento especializado comienza en edades tempranas, las posibilidades de desarrollo y calidad de vida serán más efectivas (Cuesta, Sánchez, Orozco, Valenti y Cottini, 2016).

Hay que fomentar una práctica deportiva regular, continua y progresiva. Para ello, la actividad física y deportiva debe ser entendida como un medio de desarrollo personal y social, como una fuente de salud y de ocio (Cuesta, Sánchez, Orozco, Valenti y Cottini, 2016). Además de la parte competitiva, el deporte ofrece como alternativa una práctica orientada más a lo lúdico.

Aunque las dificultades motrices no son un rasgo característico contemplado dentro del propio diagnóstico TEA, es evidente que las personas con este tipo 
de trastorno presentan problemas en el desarrollo de estas habilidades. Estas dificultades pueden manifestarse tanto en la coordinación general como en las habilidades motrices gruesas y finas (Pan, Tsai \& Chu, 2009) provocando una baja participación en actividades físicas y programas deportivos (Sowa \& Meulenbroek, 2012; Staples \& Reid, 2010).

Además del beneficio para la salud es importante tener en cuenta el impacto social que conlleva el practicar ejercicio físico. Asimismo, si la práctica deportiva es a nivel grupal, en el caso de las personas con TEA el desarrollo social que experimentarán será mucho más beneficioso. En este caso es necesario trabajar y potenciar las habilidades sociales pues resulta ser una de las áreas con mayor déficit (Villalba, 2015).

Hay que trabajar de manera constante y progresiva en la planificación y desarrollo de proyectos y programas que beneficien la educación integral de las personas con necesidades especiales. Los niños con TEA necesitan un programa integral donde se adapten sus necesidades y se potencien sus capacidades, con la finalidad de mejorar su calidad de vida y la del entorno que los rodea (Cruz, 2015). El programa deportivo puesto en marcha en este estudio sirvió para facilitar la accesibilidad a la práctica de un deporte colectivo, en este caso de cooperación y oposición, mediante un programa de entrenamiento deportivo basado en juegos predeportivos para niños con TEA.

\section{Método}

\section{Participantes}

Debido a que el programa iba dirigido a menores de edad, el proyecto deportivo debió contar con la supervisión y la aprobación del comité de ética de la institución a la que pertenecen los autores. Los participantes fueron seleccionados de manera aleatoria a partir del anuncio del programa deportivo. Las familias interesadas contactaron con el responsable del programa para concertar una entrevista y determinar el nivel de funcionamiento del niño participante. El perfil seleccionado debia cumplir con una serie de características:

- Niños y niñas con una edad comprendida entre los seis y los doce años.

- Niños y niñas con un diagnóstico TEA con un grado 1 de severidad y necesidad de apoyos

Finalmente, fueron trece los niños que participaron en el programa, con edades comprendidas entre los seis y los diez años (Gráfico 1).

Gráfico 1: Edades de los participantes

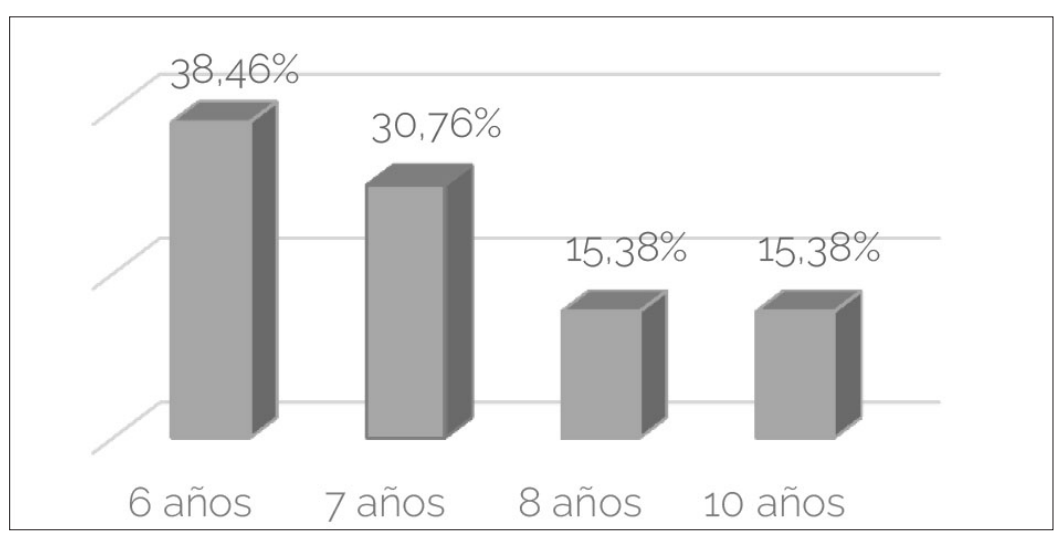

Fuente: Elaboración propia 


\section{Procedimiento}

Con el propósito de planificar y completar el programa deportivo se conformó un grupo de trece niños. El diseño de las sesiones y los juegos estuvieron supervisados por un equipo compuesto por psicólogos, maestros de educación fisica y entrenadores.

Las sesiones fueron ajustadas a unos niveles básicos propios del deporte del fútbol. Los juegos recogieron aspectos fisicos, técnicos y tácticos con la intención de aprender y comprender el deporte, y transferir los conocimientos adquiridos a otros contextos y ámbitos deportivos. Asimismo los juegos fueron adaptados individualmente para cada uno de los participantes. De esta forma, cada niño pudo participar en las actividades de acuerdo a sus características particulares.

El programa deportivo se realizó en la localidad de Alcorcón (Madrid, España) y tuvo una duración de cinco meses, comenzando en enero y finalizando en mayo. Siguiendo el calendario escolar se realizaron dos sesiones semanales de una hora de duración cada una de ellas. El espacio deportivo donde se realizaron los entrenamientos fue un gimnasio deportivo, el cual fue adaptado para realizar cada uno de los juegos diseñados. Para cada una de las sesiones se planificaron diferentes juegos deportivos sencillos y dinámicos con y sin balón, con el objetivo de modelar nuevas habilidades o de potenciar otras habilidades ya adquiridas. Estas actividades consistían en salto, pases, conducción del balón o juegos por parejas, entre otras (López Díaz et al., 2017).

\section{Instrumentos}

Se elaboró un cuestionario de treinta preguntas con el fin de conocer el estado de la cuestión referente al ocio deportivo de los niños con TEA y de medir el nivel de satisfacción del programa deportivo organizado. Este cuestionario fue diseñado Ad Hoc, tras una extensa búsqueda bibliográfica y aplicando la metodología Delphi, con un grupo de expertos en Educación Física inclusiva que contaran con entre 5 y 10 años de experiencia. El resultado final fue un cuestionario conformado por preguntas de carácter dicotómico, preguntas con varias opciones de respuesta, preguntas abiertas y preguntas tipo Likert con cuatro opciones de respuesta donde 1 era totalmente de acuerdo y 4 totalmente en desacuerdo.

El cuestionario final fue administrado a las trece familias de los niños que participaron en el Programa Deportivo y fue cumplimentado una única vez por uno de los padres o persona al cargo (padre, madre o tutor legal). El análisis de datos llevado a cabo ha sido de carácter descriptivo, haciéndose uso de datos porcentuales y estudios de frecuencias a través de gráficos en los que se cruzan las distintas variables objeto de estudio.

\section{Resultados}

Las localidades de procedencia de los participantes fueron Alcorcón (46,15\%),

Fuenlabrada (15,38\%) y Madrid-Capital (38,46\%).

Por un lado se preguntó a las familias si los niños practicaban alguna actividad deportiva. Un 53,84\% afirmó que sus hijos sí practicaban alguna actividad deportiva, mientras que el 46,15\% restante indicó que no realizaban ninguna (Gráfico 2). Un $14,28 \%$ de los que decian practicar se decantaban por el multideporte, un $42,85 \%$ 
por la natación, un 14,28\% por el judo (14,28\%), un 14,28\% por el fútbol $(14,28 \%$ ) y un $14,28 \%$ por el kárate. El $71,42 \%$ realizaba este tipo de actividad fuera del centro escolar mientras que el 28,57\% restante lo realizaba dentro del colegio.

Gráfico 2: ¿Practica alguna actividad deportiva?

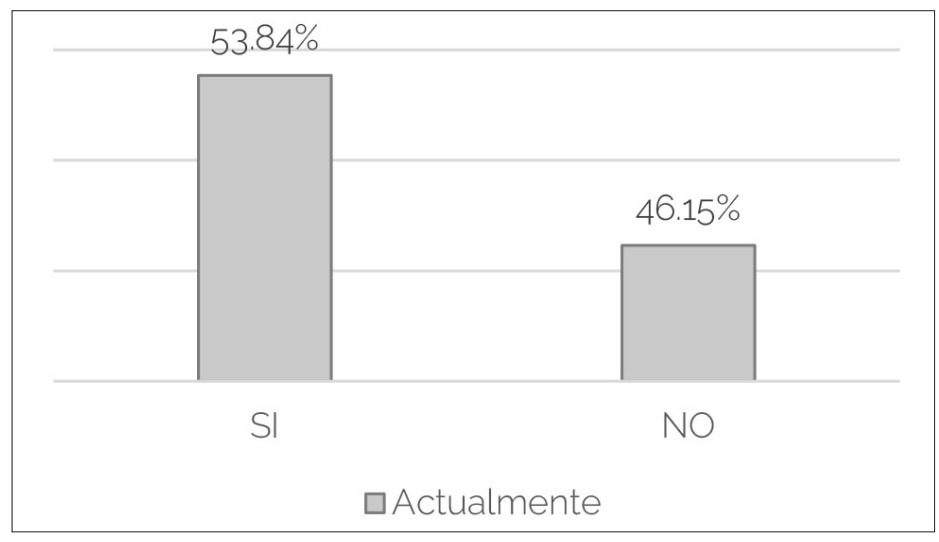

Fuente: Elaboración propia

Por otro lado también se preguntó a las familias si los niños habian realizado anteriormente algún tipo de actividad deportiva. Un 38,46\% contestó que sí, mientras que más de la mitad (61,53\%) especificó que no lo había hecho (Gráfico 3). El deporte practicado anteriormente también había sido muy variado: multideporte (20\%), kárate (20\%), patinaje (20\%), natación (20\%) y fútbol (20\%). Un 40\% lo hizo en el colegio mientras que el $60 \%$ restante lo hizo fuera del centro escolar.

Gráfico 3: ¿Ha practicado alguna actividad deportiva?

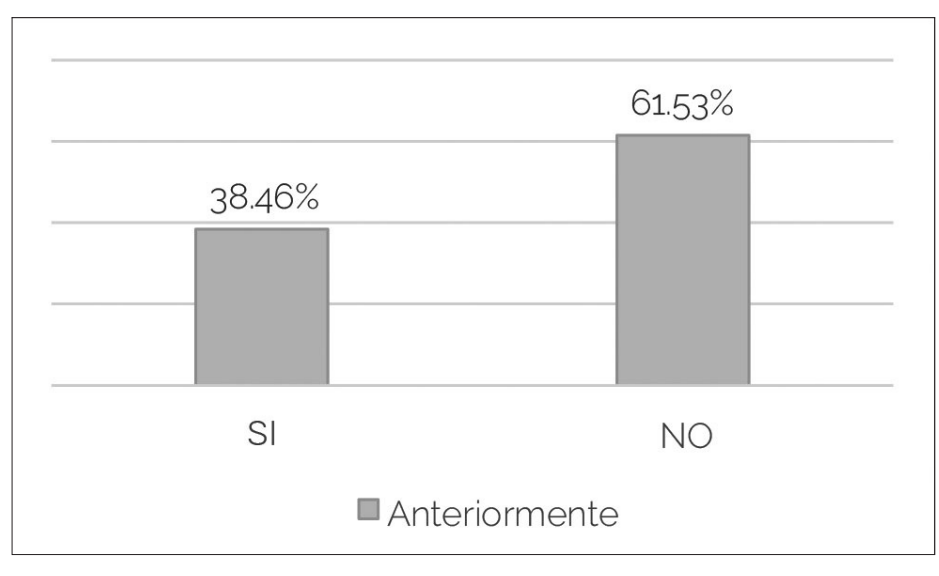

Fuente: Elaboración propia

Asimismo, del total de los niños, un 23,07\% había practicado deporte anteriormente, manteniendo actualmente la práctica deportiva aunque no el mismo deporte. Un $30,76 \%$ practicaba deporte en la actualidad, aunque no lo había hecho anteriormente. Un 15,38\% no practicó deporte aunque si había empezado a hacerlo recientemente. Por el contrario un 30,76\% nunca habia hecho ningún tipo de actividad deportiva (Gráfico 4). 


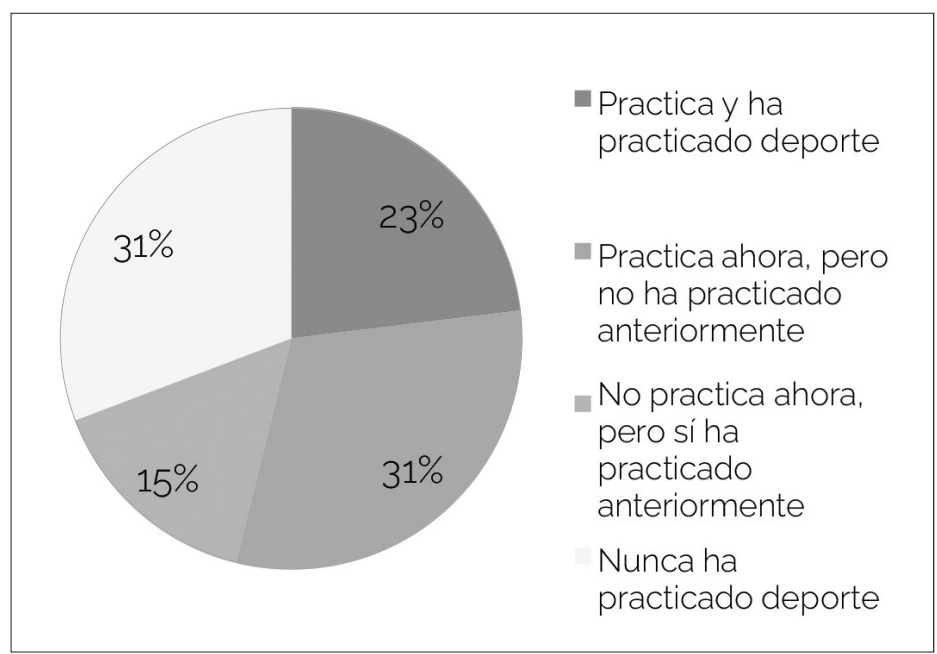

Fuente: Elaboración propia

El 100\% de las familias consideraba que el programa deportivo había sido adecuado para sus hijos. Respecto a la difusión del programa deportivo para captar niños, el $69,23 \%$ de las familias estaba totalmente de acuerdo en que ha sido suficiente y adecuada. Un 23,07\% estaba de acuerdo y solo un 7,69\% estaba en desacuerdo. El total de las familias consideraba que las reuniones mantenidas con el responsable del programa les habian servido para conocer todo el Programa Deportivo. Ese mismo porcentaje también afirmó que se habian trabajado los objetivos principales establecidos.

Los contenidos impartidos referentes a la práctica predeportiva en fútbol y a la metodología de trabajo empleada habian sido adecuados según el total de las familias. El 100\% especificó que el clima de las sesiones había sido satisfactorio para el buen desarrollo de los entrenamientos. En cuanto a los recursos y materiales empleados, el 69,23\% estuvo totalmente de acuerdo y el 30,76\% estuvo de acuerdo. Nadie mostró grado de desacuerdo alguno en lo relativo a este ítem. Respecto a la duración del programa, un 30,76\% pensó que había sido insuficiente y el 53.76\% que había sido suficiente, mientras que el resto no valoró esta cuestión (15,48\%) (Gráfico 5). Respecto a los horarios de los entrenamientos, el 84,61\% estuvo totalmente de acuerdo en que eran adecuados. Con respecto a la duración de las sesiones, sin embargo, estuvo totalmente de acuerdo un porcentaje menor, el $76,92 \%$. 


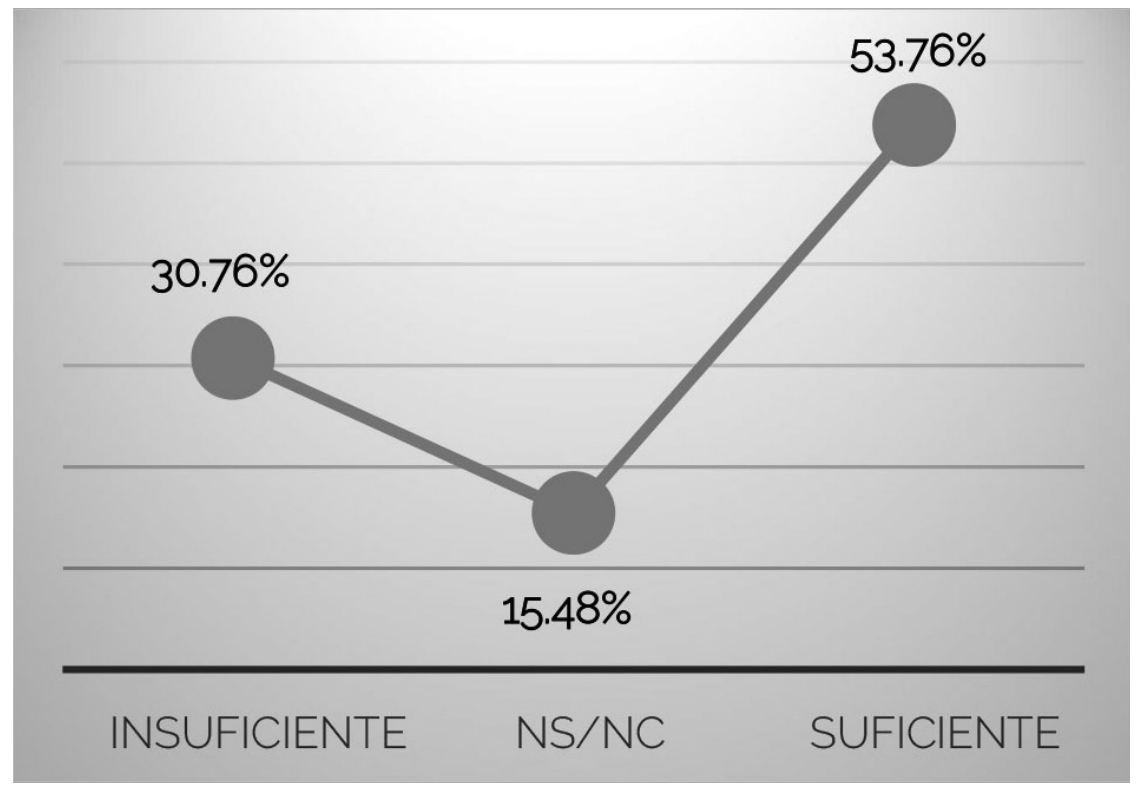

Fuente: Elaboración propia

Todas las familias consideraron que el programa habia cumplido con las expectativas, además de ser útil en el desarrollo de sus hijos. El 84,61\% de las familias consideró que su hijo había aprovechado la mayor parte de las sesiones de forma satisfactoria. El 76,92\% estuvo totalmente de acuerdo en considerar que se había producido una notable mejora en las habilidades psicomotrices y sociales de sus hijos.

El 100\% de las familias consideró la labor de los profesionales como satisfactoria y permitió el progreso de sus hijos a lo largo de las sesiones. Asimismo, todos indicaron que recomendarian este tipo de programas a otras familias que busquen actividades para sus hijos con similares caracteristicas. El 84,61\% estuvo completamente satisfecho con el Programa Deportivo y un 15.38\% estuvo satisfecho.

Respecto a las sugerencias de mejora para una posible repetición de un programa deportivo de estas características, las familias señalaron principalmente la ampliación de la duración del programa, el respeto por los horarios compatibles con el colegio, la posibilidad de ampliar los entrenamientos a otros lugares para facilitar el desplazamiento, dar una mayor difusión al programa y tener una continuidad como beneficio en la evolución de los niños.

\section{Discusión}

Existe una baja participación en actividades deportivas por parte de los niños con TEA. Esto puede deberse a las particularidades que presenta este colectivo unido a las barreras que dificultan la propia práctica deportiva (Reina, 2014). Tal y como se ha puesto de relieve y como también indican Todd \& Reid (2006), existe una baja participación del colectivo TEA en la realización de actividades físicas y deportivas debido a los problemas generados a nivel motor, las dificultades de planificación y de autocontrol y la baja motivación. Dichas dificultades de acceso a la población diana puede acrecentarse quizá debido a las dificultades para la difusión del programa. 
Como puede observarse, el hecho de que puedan realizar poca práctica deportiva normalmente va ligado al desempeño de deportes individuales. Esto puede deberse a las dificultades que presentan en habilidades sociales (Ruggieri, 2013). Se ha indicado la práctica del fútbol en algún caso puntual en el que el participante tenía desarrolladas y asentadas las cualidades fisicodeportivas y las condiciones aptas para la práctica de este deporte.

Salvo para determinados casos puntuales, el total de las familias se muestran satisfechas con el programa y con la planificación de los entrenamientos deportivos, además de resaltar positivamente las expectativas indicadas. Es evidente que el juego forma parte del desarrollo de la vida social durante los primeros años. Todo el mundo necesita jugar y practicar ejercicio físico y/o deportivo, no solo para satisfacer sus necesidades básicas sino también para el propio disfrute personal (López Díaz et al., 2017; Olayo y Moreno, 1999).

Un porcentaje mínimo (15.38\%) considera que no han aprovechado el programa completamente, lo que puede deberse a la ausencia de dias por motivos de enfermedad del niño, el tiempo disponible y la distancia del lugar u otras razones personales. Tres cuartas partes consideran que ha habido una mejora en cuanto a las habilidades psicomotrices y sociales. La práctica deportiva por parte del colectivo TEA ha sido usada en los últimos años como alternativa a otro tipo de terapias. Este tipo de medidas se utiliza con el fin de mejorar tanto en habilidades psicomotrices como en habilidades sociales (Ibáñez y Mudarra, 2004).

La duración del programa es el único punto a destacar negativamente, pues casi un tercio de las familias considera que ha sido insuficiente. Debe tenerse en cuenta que el proyecto era totalmente nuevo y que se requería de un tiempo determinado para poder ponerlo en marcha. Esto imposibilitó que pudiese desarrollarse durante todo el curso escolar. No obstante, el programa tuvo una duración de cinco meses, es decir, medio curso académico.

Hay que resaltar la creación y el beneficio que puede proporcionar este tipo de escuelas deportivas. Si se logra romper los prejuicios y barreras hacia este tipo de colectivos será más fácil trabajar con ellos, pues habrá que enseñar y modificar la conducta de estos sujetos para que entiendan el juego y puedan participar en diferentes contextos deportivos. El futuro inmediato debe ser incluir a este tipo de colectivos en cualquier escuela deportiva favoreciendo asi el logro de una verdadera inclusión.

\section{Conclusiones}

La participación en escuelas deportivas de carácter futbolístico de niños con TEA es posible si se realizan las modificaciones necesarias en los entrenamientos, de forma que el trabajo se desarrolle de manera gradual y por aproximaciones sucesivas. La participación en ellas permite conseguir una mejora en el rendimiento psicomotor de los jugadores, así como una modificación favorable de las variables relacionadas con la participación social. Se genera, de esta forma, una oferta de actividad deportiva o de ocio que, a pesar de haber sido poco estudiada en la bibliografia cientifica, supone un incremento en la posibilidad de participar en actividades comunes al grupo social de referencia de los participantes. En este caso, las familias participantes son quienes han señalado este tipo de programas como una oportunidad para que sus hijos sigan creciendo y mejorando tanto a nivel social como a nivel motor, y por ende, a nivel personal. 
Aunque resulta necesario profundizar en este trabajo contando con una mayor muestra -la cual resulta de dificil acceso también por la heterogeneidad individual en la constitución de los grupos-, los resultados obtenidos son prometedores en cuanto a la consecución de objetivos.

Nota:

Aprobación final del artículo:editora responsable Mag. Verónica Zorrilla de San Martín.

Contribución de autoría: el autor principal, José María López Díaz, ha desarrollado y ejecutado la implementación del programa experimental incluyendo la evaluación y selección de participantes, controlando las variables metodológicas necesarias para garantizar el mayor rigor académico en la puesta en marcha del trabajo. El segundo autor, Ricardo Moreno Rodriguez, ha coordinado el desarrollo del trabajo global, participado en el diseño del proyecto, elaboración y ajuste de pruebas de evaluación, ajuste de las sesiones y actividades comprendidas en las mismas, asi como soporte en la fase documental del trabajo. El tercer autor, José Luis López Bastías, actuó como evaluador de los participantes, participó en el diseño de las actividades y en el ajuste de las mismas a las diferentes capacidades de los participantes, asi como en el diseño didáctico de las actividades. 


\section{Bibliografía}

AMERICAN PSYCHIATRIC ASSOCIATION (2013). Diagnostic and statistical manual of mental disorders, fifth edition (DSM-5). Washington DC: APA

AMERICAN PSYCHIATRIC ASSOCIATION (2000). Diagnostic and statistical manual of mental disorders.

CONFEDERACIÓN AUTISMO ESPAÑA (2003). Autismo, calidad de vida. Madrid: Confederación Autismo España e Instituto de Migraciones y Servicios Sociales (IMSERSO).

CRUZ, C. (2015). Natación adaptada. Una alternativa de integración para niños con autismo en Cuba. Revista Acción, 12. No 23. http://accion.uccfd.cu/public/ journals/2/accionhtml/issues/Vol_13_No_23/files/10.pdf

CUESTA, J. L., SÁNCHEZ, S., OROZCO, M. L., VALENTI, A. \& COTTINI, L. (2016). Trastorno del espectro del autismo: intervención educativa y formación a lo largo de la vida. Psychology, Society, \& Education. Vol. 8(2), pp. 157-172 https:// dialnet.unirioja.es/servlet/articulo? codigo $=6360233$

DICICCO-BLOOM, E., LORD, C., ZWAIGENBAUM, L., COURCHESNE, E., DAGER, S. R., SCHMITZ, C., SCHULTZ, R. T., CRAWLEY, J., \& YOUNG L. J. (2006). The developmental neurobiology of autism spectrum disorder. [La neurobiología del desarrollo del trastorno del espectro autista]. Journal of Neuroscience, 26: 6897-6906. https://doi.org/10.1523/JNEUROSCl.1712-06.2006

FRITH, U. (1989). Autism: Explaining the enigma. Oxford: Blackwell. (Traducción al castellano: FRITH, U. (1992). AUTISMO: HACIA UNA EXPLICACIÓN DEL ENIGMA. MADRID: ALIANZA).

GONZÁLEZ, H.V., Y SOSA, B. S. (2008). Importancia de la práctica de deportes en personas discapacitadas para lograr una mejor calidad de vida. En Contribuciones a las Ciencias Sociales. https://www.eumed.net/rev/cccss/02/gcss.htm

GUTIÉRREZ, M. (2004). El valor del deporte en la educación integral del ser humano. Revista de educación. (335). pp. 105-126. http://www.revistaeducacion. educacion.es/re335/re335_10.pdf

IBÁÑEZ, P. Y MUDARRA, M. J. (2004). Relaciones sociales de personas con discapacidad, en el ocio y tiempo libre. Revista Española de Pedagogía, 229. pp. 521-540. https://dialnet.unirioja.es/servlet/articulo?codigo=1051463

JULIÁN, J., ABARCA-SOS, A., MURILLO, B. Y AIBAR, A. (2015). Secuenciación de juegos populares en educación fisica con alumnado diagnosticado dentro del Trastorno del Espectro Autista. Revista Tándem, Didáctica de la Educación Física, 47. pp. 65-74.

KRUGER, G., SILVEIRA, S. \& MARQUES, A. (2019). Motor skills of children with autism spectrum disorder. IHabilidades motoras en niños con trastorno del espectro autista]. Revista Brasileira de Cineantropometria y Desempenho Humano, 21, pp. 1-8. https://dx.doi.org/10.1590/1980-0037.2019v21e60515 
LÓPEZ díAZ, J. M., MORENO-RODRIGUEZ, R., ALCOVER, C. M., GARROTE, I., \& SÁNCHEZ, S. (2017). Effects of a program of sport schools on development of social and psychomotor skills of people with autistic spectrum disorders: A pilot project. [Efectos de un programa de escuelas deportivas en el desarrollo de las habilidades sociales y psicomotoras de personas con trastornos del espectro autista: un proyecto piloto]. Journal of Education and Training Studies, 5(8), pp. 167-177. https://eric.ed.gov/?id=EJ1150538

OKELY, A. D., BOOTH, M. L., \& PATTERSON, J. W. (2001). Relationship of physical activity to fundamental movement skills among adolescents. [Relación de la actividad física con las habilidades fundamentales de movimiento entre los adolescentes]. Medicine and science in sports and exercise, 33(11). pp. 1899-1904. DOl: 10.1097/00005768-200111000-00015.

OLAYO, J. M. (AUTOR), MORENO, F. (COORD.) (1999). El alumnado con discapacidad, juegos y deportes especificos (II). La actividad fisica y deportiva extraescolar en los centros educativos. Ministerio de Educación y Cultura. Ed. Secretaría General Técnica, Centro de Publicaciones.

PAN C-Y, TSAI C-L \& CHU C-H. (2009). Fundamental Movement Skills in Children Diagnosed with Autism Spectrum Disorders and Attention Deficit Hyperactivity Disorder. [Habilidades fundamentales de movimiento en niños diagnosticados con Trastornos del Espectro Autista y Trastorno por Déficit de Atención con Hiperactividad]. Journal of Autism and Developmental Disorders; 39(12). pp. 16941705. https://doi.org/10.1007/s10803-009-0813-5

REINA, R. (2014). Inclusión en deporte adaptado: dos caras de una misma moneda. Psychology, Society \& Education, 6(1), pp. 55-67. http://ojs.ual.es/ojs/index.php/ psye/article/view/508/486

RIVIĖRE, A. (1998). El tratamiento del autismo como trastorno del desarrollo: principios generales. En A. Rivière y J. Martos (Coord.) El tratamiento del autismo. Nuevas perspectivas (pp. 23-60). Madrid: Asociación de Padres de Personas con Autismo (APNA) e Instituto de Migraciones y Servicios Sociales (IMSERSO).

RUGGIERI, V. L. (2013). Empatía, cognición social y trastornos del espectro autista. Revista de Neurología, 56(1). pp. 13-21. https://www.amece.es/images/ autismo11.pdf

RUIZ, D., SALINERO, J. J., GONZÁLEZ, C., LLEDÓ, M., GARCIA, T. YTHEIRS, C. I. (2015). Descripción de la práctica de actividad física, habilidades motrices básicas y composición corporal en niños y jóvenes de espectro autista. Diferencias por sexo. Retos, 28, pp. 61-65. https://doi.org/10.47197/retos.voi28.34817

SALLIS, J. F., PROCHASKA, J. J. \& TAYLOR, W. C. (2000). A review of correlates of physical activity of children and adolescents. [Una revisión de los correlatos de la actividad fisica de niños y adolescentes]. Medicine and Science in Sports and Exercise, 32(5), pp. 963-975. https://doi.org/10.1097/00005768-200005000$\underline{00014}$

SOWA, M. \& MEULENBROEK, R. (2012). Effects of physical exercise on Autism Spectrum Disorders: A meta-analysis. [Efectos del ejercicio fisico en el Trastorno del Espectro Autista: un meta-análisis]. Research in Autism Spectrum Disorders, 6(1) pp. 46-57. https://doi.org/10.1016/j.rasd.2011.09.001 
STAPLES, K., \& REID, G. (2010). Fundamental Movement Skills and Autism Spectrum Disorders. [Habilidades fundamentales de movimiento y Trastornos del espectro autistal Journal of Autism and Developmental Disorders; 40(2), pp 209-217. https://doi.org/10.1007/s10803-009-0854-9

TODD, T. \& REID, G. (2006). Increasing Physical Activity in Individuals With Autism. [Aumento de la actividad física en personas con autismo]. Focus on autism and other developmental disabilities, 21(3), pp 167-176. https://doi.org/10.1177/10883 576060210030501

VILLALBA, I. M. (2015). Un proyecto de ocio y actividad física para personas con Trastornos del Espectro Autista (TEA): coopedaleando. Revista pedagógica, 28, pp 167-182. https://dialnet.unirioja.es/servlet/articulo?codigo=5323359

WONG, C., ODOM, S., HUME, K., COX, A., FETTIG, A., KUCHARCZYK, S., BROCK, M., PLAVNICK, J., FLEURY, V. \& SCHULTZ, T. (2015). Evidence based practices for children, youth, and young adults with autism spectrum disorder: a comprehensive review [Prácticas basadas en evidencia para niños, jóvenes y adultos jóvenes con trastorno del espectro autista: una revisión integral]. Journal of Autism and Developmental Disorders, 45. pp 1951-1966. https://doi. org/10.1007/s10803-014-2351-Z 